Check for updates

Cite this: RSC Adv., 2017, 7, 33378

\title{
Tumor homing and penetrating peptide- conjugated niosomes as multi-drug carriers for tumor-targeted drug delivery $\dagger$
}

\author{
Didem Ag Seleci, (D) Muharrem Seleci, Frank Stahl* and Thomas Scheper
}

\begin{abstract}
The development of nanoscale drug delivery systems, which can mediate efficient tumor targeting together with high cellular internalization, is crucial for glioma treatment. The combination of therapeutic agents in nanoparticles provides synergistic effects and allows further surface modifications with targeting ligands for specific glioma therapy. To achieve this goal, both doxorubicin and curcumin were encapsulated in polyethylene glycolated niosomes (PEGNIO). The surface of co-drug loaded PEGNIO was modified with tumor homing and penetrating peptide (tLyp-1). Physicochemical properties were determined via dynamic light scattering (DLS) and spectral analysis. Moreover, flow cytometry studies were performed to examine the specific cellular uptake of the tLyp-1 targeted niosomal formulation. In vitro cytotoxicity and inhibition of tumor-like spheroids growth were investigated on human glioblastoma (U87) and human mesenchymal stem cells (hMSC) cells. The results clearly indicated that the strategy by co-administration of doxorubicin and curcumin with thyp-1 functionalized niosomes could significantly improve antiglioma treatment.
\end{abstract}

Received 5th May 2017

Accepted 21st June 2017

DOI: 10.1039/c7ra05071b

rsc.li/rsc-advances compared to their corresponding native drugs in $2 \mathrm{D}$ and $3 \mathrm{D}$ culture. ${ }^{6}$ In another study $\mathrm{Xu}$ et al. studied in vitro cytotoxicity effects of paclitaxel and temozolomide co-loaded in polymer nanocomposites and the results suggested that the composite gel possessed much higher growth-inhibiting effects as well as apoptosis-inducing rates in glioma cells than other formulations. ${ }^{\mathbf{8}}$

Niosomes are drug carriers exhibiting a bilayer structure and are in most cases formed by self-association of non-ionic surfactants and cholesterol in aqueous phase. They can accommodate a large number of drugs with a wide range of solubilities. ${ }^{9,10}$ Hydrophilic drugs and lipophilic drugs can be entrapped into the aqueous core and membrane bilayer of niosome respectively. ${ }^{\mathbf{1 1}, \mathbf{1 2}}$ Therefore, niosomes are promising nanocarriers in multi-drug delivery applications. The efficiency of niosomal multi-drug delivery systems can be further improved by active targeting for tumor therapy by using a ligand coupled to the surface of niosomes. In this way, nanocarriers can be actively taken up, for example, via receptor-mediated endocytosis. ${ }^{13}$ Recently, Tavano et al. reported the dual encapsulation of hydrophobic curcumin or quercetin and hydrophilic doxorubicin in Pluronic-based niosomes for cancer multi-drug delivery. ${ }^{14}$ Besides, the surface of the niosomes was coupled with transferrin and/or folic acid for breast cancer targeting. Results showed the high potential of the dual drug loaded niosomes in breast tumor treatment.

Neuropilin-1 (NRP-1) is a transmembrane protein overexpressed on the surface of both glioma and endothelial cells of angiogenic blood vessels. ${ }^{\mathbf{1 5 - 1 7}}$ CGNKRTR (tLyP-1) is a homing peptide which penetrates tumor cell through an NRP-1
Institute of Technical Chemistry, Leibniz University of Hannover, Callinstr. 5, 30167 Hannover, Germany. E-mail: stahl@iftc.uni-hannover.de

$\dagger$ Electronic supplementary information (ESI) available. See DOI: 10.1039/c7ra05071b 
mediated endocytosis via C-end Rule (CendR) internalization pathway. ${ }^{18,19}$ Therefore, tLyP-1 is a promising targeting ligand for the delivery of therapeutic agents to tumors. $\mathrm{Xu}$ et al. produced tLyp-1 targeted camptothecin-loaded mesoporous silica nanoparticles and it showed minimal adverse effects on human mesenchymal stem cells (hMSC), whereas significant induction of tumor cell death was observed. ${ }^{20}$ Moreover, $\mathrm{Hu}$ et al. conjugated the tLyp-1 peptide to the surface of paclitaxel (PTX) loaded PEG-PLA nanoparticles via a maleimide-thiol coupling reaction for anti-glioma drug delivery. They achieved the longest survival of the mice bearing intracranial C6 glioma treated with PTX-loaded tLyp-1-nanoparticles in comparison to PTX loaded nanoparticles. ${ }^{17}$

In this study, polyethylene glycolated niosomes (PEGNIO) were prepared from span60, cholesterol and 1,2-distearoyl-snglycero-3-phosphoethanolamine- $N$-[maleimide (polyethylene glycol)-2000] (DSPE-PEG (2000) Maleimide) for multi-drug delivery. Curcumin (C) and doxorubicin (D) were encapsulated into the PEGNIO. The tumor-homing peptide tLyp-1 was conjugated to $\mathrm{C}$ and $\mathrm{D}$ encapsulated PEGNIO (PEGNIO/D-C) via the formation of a thioether linkage. The effect of the PEGNIO/ D-C/tLyp-1 on human glioblastoma cells (U87) and hMSC was investigated in detail. Our results suggest that this targeted and co-loaded drug delivery platform could improve the efficacy of doxorubicin on glioma therapy.

\section{Materials and methods}

\section{Materials}

DSPE-PEG(2000) Maleimide was provided by Avanti (Alabama, USA). Sorbitan monostearate (Span60), cholesterol, 3-(4,5dimethylthiazol-2-yl)-2,5-diphenyl tetrazoliumbromide (MTT), Dulbecco's Modified Eagle Medium (DMEM), curcumin and rhodamine 6G (Rh6g) were ordered from Sigma Aldrich (Munich, Germany). Alpha Minimum Essential Media (AlphaMEM) was purchased from Life Technologies (Darmstadt, Germany). Doxorubicin hydrochloride was purchased from Cayman Chemical (Michigan, USA). tLyP-1 peptide (CGNKRTR) was ordered from GeneCust (Ellange, Luxembourg).

\section{Preparation of niosomes}

The thin film hydration method was used to prepare PEGylated niosomes. ${ }^{21}$ Span 60, cholesterol and DSPE-PEG(2000) Maleimide were dissolved in $1.0 \mathrm{~mL}$ chloroform in a round-bottom flask with the $\mathrm{mM}$ ratio $(4.95: 4.95: 0.1)$. The solvent was evaporated with constant rotation under reduced pressure to form a thin lipid film. Doxorubicin or Rh6g loaded niosomes were obtained by hydrating the thin lipid film with $1.0 \mathrm{~mL}$ of doxorubicin or Rh6g $\left(0.22 \times 10^{-3}\right.$ and $0.42 \times 10^{-3} \mathrm{M}$, respectively) aqueous solution at $60{ }^{\circ} \mathrm{C}$ for $60 \mathrm{~min} \cdot{ }^{14,22}$ To obtain doxorubicin-curcumin-loaded niosomes, $200 \mu \mathrm{L}$ of curcumin solution $\left(2.18 \times 10^{-3} \mathrm{M}\right)$ was added to the initial chloroform mixture. ${ }^{14}$ After vortexing, the solvent was evaporated under reduced pressure, and the obtained film was then hydrated with $1.0 \mathrm{~mL}$ of doxorubicin aqueous solution at $60{ }^{\circ} \mathrm{C}$ for $60 \mathrm{~min}$. Afterward, the niosomal solution was equilibrated at room temperature overnight to complete annealing and partitioning of the drug between the lipid bilayer and the aqueous phase. ${ }^{23}$ Small unilamellar vesicles (SUVs) were prepared starting from multilamellar vesicles (MLVs) by sonication in an ultrasonic bath and fallowing extruding the niosomes through $0.4 \mu \mathrm{m}$ and $0.1 \mu \mathrm{m}$ pore size polycarbonate filters (mini-extruder set Avanti polar lipids, sample volume $1.0 \mathrm{~mL}$ ).

Niosomes were purified by a flow of niosome suspensions across a Sephadex G-25 gel column and volume of eluted niosomes was adjusted to $1.0 \mathrm{~mL}$.

\section{Preparation and characterization of tLyp- 1 conjugated niosomes}

For the preparation of tLyp- 1 targeted niosomes, the thiol group of tLyp-1 was coupled with the maleimide group of PEG chains on niosomes. tLyp-1 peptide was dissolved in $50 \mathrm{mM}$ HEPES buffer, $\mathrm{pH} 6.5$ at $200 \mu \mathrm{g} \mathrm{mL} \mathrm{mL}^{-1}$ concentration $(50 \mu \mathrm{L})$ were reacted with niosomes $(950 \mu \mathrm{L})$ for overnight at room temperature resulting in the formation of a thioether linkage. ${ }^{24,25}$ The products were then purified using a $14 \mathrm{kDa}$ dialysis bag to remove the unconjugated peptides. Schematic representation of co-drug encapsulation and the bioconjugation processes are shown in Scheme 1.

The size of the niosomes were measured by dynamic light scattering (DLS) analysis using Malvern Zetasizer Nano ZSP. The polydispersity index (PDI) was used as a measure of the width of size distribution. Each sample was measured three times. The morphology of large unilamellar PEGNIO (before extrusion) was monitored via Olympus BX41 upright microscope. 100X immersion oil objective was used.

To estimate the tLyp-1 conjugation efficiency, BCA Protein Assay was used. ${ }^{4} 25 \mu \mathrm{L}$ of BSA standards, PEGNIO (as blank) and PEGNIO/tLyp-1 were added in triplicate wells (96-well plate) and $200 \mu \mathrm{L}$ of BCA Protein Assay Reagent were added to the samples. After incubation at $37{ }^{\circ} \mathrm{C}$ for $30 \mathrm{~min}$, the absorption was measured via microplate reader (BioTek Epoch) at $562 \mathrm{~nm}$. Conjugation efficiency was expressed as the percentage of the peptide bound to the surface of noisome, referred to the amount of peptide that is present initially.

\section{Stability}

The stability of PEGNIO/D-C/tLyp-1 was tested via DLS analysis. PEGNIO/D-C/tLyp- 1 was stored at $4{ }^{\circ} \mathrm{C}$ in the dark. The particle size and PDI values were measured after 30 days. Moreover, the

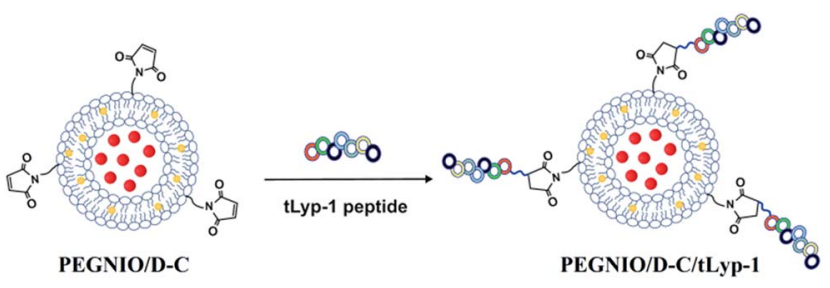

Scheme 1 Schematic representation of co-drug encapsulation and the bioconjugation process (yellow dots: curcumin, red dots: doxorubicin). 
particle size of PEGNIO/D-C/tLyp-1 was measured in cell culture media after the incubation at $37^{\circ} \mathrm{C}$ for $24 \mathrm{~h}$.

\section{Entrapment efficiency}

After encapsulation, the D-C loaded niosomes were purified using Sephadex G-25 gel column. The percent of encapsulation efficiency $(E \%)$ was expressed as the percentage of the drug entrapped in niosomes (and thus not removed via Sephadex column) referred to the initial amount of drug that is present in the non-purified sample (eqn (1)). $200 \mu \mathrm{L}$ of non-purified and purified niosomes diluted in $600 \mu \mathrm{L}$ of methanol and niosomes were separated by centrifugation at $12000 \mathrm{rpm}$ for $5 \mathrm{~min}$. This step allows the breaking of niosomal membranes and the release of the encapsulated drug. A calibration curve was derived with a known concentration of free doxorubicin by fluorescence emission measurements at $595 \mathrm{~nm}$ using NanoDrop 3300. The stock solutions of doxorubicin were prepared at $1.0 \mathrm{mg} \mathrm{mL}{ }^{-1}$ in methanol and further diluted with methanol in the concentration range $1.0-50 \mu \mathrm{g} \mathrm{mL}^{-1}$. The amount of doxorubicin in purified and non-purified samples was calculated according to the calibration curve $\left(y=850.94 x+12.092, R^{2}=\right.$ 0.9973 ) via measuring fluorescence emission at $595 \mathrm{~nm}$. The limit of detection (LOD) and limit of quantification (LOQ) for doxorubicin were found to be $4.92 \mu \mathrm{g} \mathrm{mL}{ }^{-1}$ and $14.92 \mu \mathrm{g} \mathrm{mL}^{-1}$ respectively, based on $3.3 \sigma /$ slope and $10 s \sigma /$ slope formulations. The amount of curcumin in purified and non-purified samples was calculated according to the calibration curve $(y=0.1459 x+$ $0.0058, R^{2}=1, \mathrm{LOD}=0.15 \mu \mathrm{g} \mathrm{mL}{ }^{-1}$ and LOQ $=0.46 \mu \mathrm{g} \mathrm{mL}{ }^{-1}$ ), which was established with a known concentration of free curcumin $\left(0.5-10 \mu \mathrm{g} \mathrm{mL}^{-1}\right)$ by absorbance measurements at $426 \mathrm{~nm}$ using a microplate reader (BioTek Epoch).

$$
E(\%)=\frac{\text { amount of drug in purified sample }}{\text { amount of drug in non-purified sample }} \times 100
$$

\section{Drug release}

The in vitro release profiles of curcumin and doxorubicin from PEGNIO/D-C/tLyp-1 were studied using a dialysis method. PEGNIO/D-C/tLyp-1 solutions were prepared and transferred into a dialysis membrane tubing (Thermo, Slide-ALyzer MINI Dialysis Devices, 10k MWCO). The tubing was immersed in 10 $\mathrm{mL}$ of the PBS buffer ( $\mathrm{pH} 5.6$ and 7.4) containing Tween 80 $(1.0 \%, \mathrm{v} / \mathrm{v})$, placed in an incubator at $37{ }^{\circ} \mathrm{C}$ and stirred at $100 \mathrm{rpm}$. Here, Tween 80 was added to obtain optimal release conditions since curcumin has limited solubility in PBS. ${ }^{26}$ At predetermined time intervals, $0.5 \mathrm{~mL}$ samples were removed from the release medium and replaced with the same volume of fresh buffer. The amount of released doxorubicin and curcumin was calculated as \% according to the calibration curves and in respect to loading concentration (the drug concentration in niosomes, before starting the release). They were established with a known concentration of free doxorubicin and free curcumin by fluorescence emission measurements at $595 \mathrm{~nm}$ using NanoDrop 3300 and absorbance measurements at $426 \mathrm{~nm}$ using a microplate reader respectively.

\section{Cell culture}

U87 cell lines were provided from German Collection of Microorganisms and Cell Cultures (DSMZ). U87 cells were grown in DMEM containing 10\% fetal calf serum (FCS) (Biochrom $\mathrm{GmbH}$, Germany) and $1.0 \%$ penicillin/streptomycin (P/ S). Human adipose-derived mesenchymal stem cells (hMSCs) were isolated from subcutaneous adipose tissues of 3 different patients scheduled for abdominoplasty after obtaining informed written consent, as approved by the Institutional Review Board, project \#2251-2014 on 15th May, 2014. The isolated populations have been extensively characterized as mesenchymal stem cells by surface marker analysis and functional properties (differentiation capacity). hMSCs were cultured in alpha-MEM including 10\% human serum (HS) (HS, c.c.pro $\mathrm{GmbH}$, Germany) and $0.5 \%$ gentamicin. Both cell lines were cultivated and incubated with samples at $37{ }^{\circ} \mathrm{C}$ in a humidified environment with $5.0 \% \mathrm{CO}_{2}$.

\section{Cellular uptake}

Rh6g was used as a model fluorescent dye and encapsulated into niosomes. The uptake of the $\mathrm{Rh} 6 \mathrm{~g}$ loaded niosomal formulations by U87 and hMSC cells was analyzed by flow cytometry. The cells $\left(4 \times 10^{4}\right)$ were treated with PEGNIO/Rh6g and PEGNIO/Rh6g/tLyp-1 for $2 \mathrm{~h}$ and treated cells were washed two times with PBS, and then analyzed in a BD Accuri C6 cytometer.

\section{Cytotoxicity}

The cytotoxic effects of niosomal formulations and free drugs were tested on U87 and hMSC cells using MTT assay. Cells $(8 \times$ $10^{3}$ ) were seeded out in 96-well tissue plates (Sarstedt, USA) in a volume of $200 \mu \mathrm{L}$ and cultivated for three days. After this cultivation time, cells were washed once with PBS and treated with PEGNIO/D-C, PEGNIO/D-C/tLyp-1 and free D-C for $24 \mathrm{~h}$. The equivalent concentration of free doxorubicin and curcumin was used in niosomal formulations. Then the samples were removed and cells were incubated in $110 \mu \mathrm{L}$ per well $10 \%$ MTT solution (5.0 mg mL $\mathrm{mL}^{-1}$ in PBS) in the medium for $4 \mathrm{~h}$. During this incubation time, formazan complex was produced by the cells. $100 \mu \mathrm{L}$ SDS solution ( $1.0 \mathrm{~g}$ SDS in $10 \mathrm{~mL} 0.01 \mathrm{M} \mathrm{HCl}$ ) were added to each well to release the purple colored salt from the cells. After $24 \mathrm{~h}$ of incubation, UV-vis absorption was measured at $570 \mathrm{~nm}$ to $630 \mathrm{~nm}$ as the reference wavelength using a microplate reader Epoch Biotek. Besides, IC50 values (the drug concentration required for $50 \%$ inhibition of cell viability) were calculated for U87 cells using the growth sigmoidal/dose response function of Origin software.

\section{Effect on tumor-like multicellular spheroids}

The effect of niosomal formulations was tested on 3D spheroid cultures. U87 cells $\left(8 \times 10^{3}\right.$ per well $)$ in $100 \mu \mathrm{L}$ of culture medium were seeded into 96-well round-bottom ultra low attachment plates (Sarstedt, Germany) and incubated for 2 days to form spheroids. Afterward, $100 \mu \mathrm{L}$ of each sample (D-C, PEGNIO/D-C, PEGNIO/D-C/tLyp-1) was added to the spheroids 
and incubated further for 2, 4 and 6 days. Bright-field images of U87 tumor-like spheroids treated with the samples were taken with Olympus IX50 inverted light microscope equipped with an Olympus camera (SC30, Japan) by using cellSens Standard software (Olympus Co. Japan).

\section{Statistical analysis}

Statistical data analysis was performed using the Student's $t$ test. The difference between two groups was considered significant when the $p$ value was less than 0.05 .

\section{Results and discussion}

\section{Characterizations of niosomal formulations}

The morphology of large unilamellar PEGNIO was monitored using an upright microscope (Fig. S1†). Fig. S1† indicated that PEGNIOs were spherical and homogeneous in shape. The mean diameters of empty and co-drug-loaded niosomal formulations, along with the corresponding polydispersity index (PDI) and drug entrapment efficiency $(E \%)$ values, are listed in Table 1. The empty vesicle size was analyzed to be $150.3 \mathrm{~nm}$. When doxorubicin and curcumin were co-encapsulated, a slightly smaller hydrodynamic diameter as $144.1 \mathrm{~nm}$ was obtained. In our previous study we demonstrated, that the encapsulation of doxorubicin in PEGNIO did not resulted in an alteration of niosomal size. ${ }^{22}$ However, when a hydrophobic drug is encapsulated in the colloidal system, they were claimed to lead to $\mathrm{H}$ bonding between their hydroxyl groups and niosomal matrices, resulting in an increase in the niosomal cohesion and then a decrease in the diameter. ${ }^{\mathbf{1 4}}$ Here, the decrease in the size can be attributed to the entrapment of hydrophobic curcumin in the bilayer. After the conjugation of tLyp-1, the size of the niosomes did not change significantly. The size was measured to be $146.1 \mathrm{~nm}$ for PEGNIO/D-C/tLyp-1. PDI less than 0.3 corresponds to a homogeneous population for colloidal systems. ${ }^{27}$ PDI ranged from 0.175 to 0.140 for the niosomal formulations, demonstrating that the vesicle population is relatively homogeneous in size. The entrapment efficacy $(E \%)$ was calculated to be $32.6 \pm 1.9 \%$ for curcumin and $23.3 \pm 1.6 \%$ for doxorubicin.

Under the determined experimental conditions, the t-Lyp-1 peptide conjugation efficiency was calculated to be $23.9 \%$.

The stability of PEGNIO/D-C/tLyp-1 was tested via DLS analysis and no changes were observed in the size and PDI values after 30 days storage at $4{ }^{\circ} \mathrm{C}$ in the dark (data not shown). Furthermore, PEGNIO/D-C/tLyp-1 was diluted in cell culture media and was incubated at $37{ }^{\circ} \mathrm{C}$ for 24 hours. The size of the sample was measured and after incubation and no changes were observed.

\section{Drug release}

Prolonged drug retention and sustained drug release are important properties for nanoscale drug delivery systems that will minimize side effects of the drug. ${ }^{22}$ The unique structure of niosomes allows to control the release of the encapsulated drug combinations to increase antitumor activity. ${ }^{14}$ The release of doxorubicin and curcumin from PEGNIO/D-C/t-Lyp-1 was investigated using dialysis methods at $\mathrm{pH}$ 7.4, which was chosen in accordance with physiological conditions and in an acidic environment ( $\mathrm{pH}$ 5.6) similar to the tumor. The release profiles of doxorubicin and curcumin from PEGNIO/D-C/t-Lyp1 showed the faster release of both drugs under acidic environments than that at neutral pH (Fig. 1). Within $32 \mathrm{~h}$, the release of doxorubicin and curcumin was $74 \pm 1.2 \%$ and $62 \pm$ $0.6 \%$ at $\mathrm{pH} 5.6$ respectively. At physiological $\mathrm{pH}$, the release of curcumin and doxorubicin was $36 \pm 1.7 \%$ and $68 \pm 2.9 \%$. According to results, this conjugate is expected to be a promising co-drug delivery system for the tumor-targeted therapy.

\section{Cellular uptake}

To test the specificity of t-Lyp-1 targeted niosomes, Rh6g was encapsulated in PEGylated niosomes and further t-Lyp-1 was conjugated to PEG chains on niosomes. Flow cytometry was used to investigate the Rh6g uptake by hMSC and U87 cells to evaluate receptor-mediated cell targeting. The cells were treated with PEGNIO/Rh6g for $2 \mathrm{~h}$. Untreated control cells and treated cells were analyzed using a BD Accuri C6 flow cytometer. No differences were observed between targeted and non-targeted

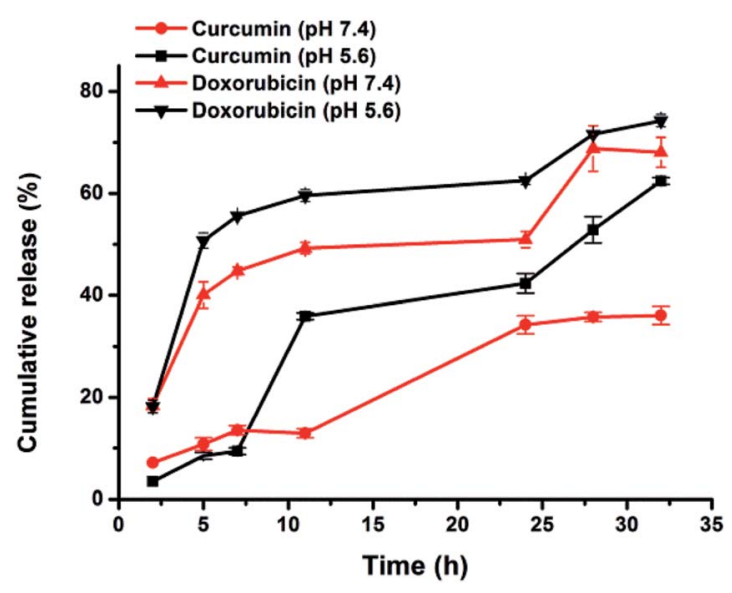

Fig. 1 In vitro cumulative release of doxorubicin and curcumin from PEGNIO/D-C/t-Lyp-1 at pH 7.4 and 5.6.

Table 1 Characterization of PEGNIO formulations

\begin{tabular}{llll}
\hline Samples & $\begin{array}{l}\text { Size }(\mathrm{nm}) \text { intensity } \\
(\%)(\text { mean } \pm \mathrm{SD})\end{array}$ & $\begin{array}{l}\text { Poly-dispersity } \\
\text { index (PDI) }\end{array}$ & Entrapment efficiency $E \%$ \\
\hline PEGNIO & $150.3 \pm 51$ & 0.175 & - \\
PEGNIO/D-C & $144.1 \pm 61$ & 0.152 & D:23.3 \pm 1.6, C:32.6 \pm 1.9 \\
PEGNIO/D-C/tLyp-1 & $146.1 \pm 69$ & 0.140 & D:22.0 \pm 1.5, C:31.2 \pm 1.8
\end{tabular}


niosomal formulations treated hMSC (Fig. 2a). In the case of U87 cells, the uptake of PEGNIO/Rh6g/tLyp-1 was higher than PEGNIO/Rh6g (Fig. 2b). Fig. 2c indicates that PEGNIO/Rh6g/ tLyp-1 was uptaken by U87 cells specifically. tLyP-1 is able to selectively home in and penetrate into tumor cells mediated NRP-1 receptor which is overexpressed in tumor cells. ${ }^{20,28}$ The expression of NRP-1 receptor, on the surface of the U87 cells would affect the enhanced cellular uptake of PEGNIO/Rh6g/ tLyp-1.

\section{Cytotoxicity}

Doxorubicin is classified as a topoisomerase-2 inhibitor and one of the most extensively used broad-spectrum anticancer drugs. However, it has some limitations in clinical use. Long treatment durations cause the development of resistance by tumor cells and toxicity for healthy tissues. Therefore, combinations of two or more agents have been used to overcome toxicity and other side effects of doxorubicin..$^{29,30}$ Curcumin's therapeutic characteristics have been demonstrated against a wide range of cancers. ${ }^{31-33}$ The major drawback of curcumin is its poor solubility and stability in water. Thus, here doxorubicin and curcumin were encapsulated in PEGNIO and further the surface of niosome was modified with t-Lyp-1 peptide.

After confirming the NRP-1 receptor-mediated glioma cell binding efficiencies of the PEGNIO/Rh6g/tLyp-1 via flow cytometry, MTT assays were performed to evaluate relative abilities of the various niosomal formulations in inhibiting growth of tumor (U87) and non-cancer (hMSC) cells (Fig.3). PEGNIO/D-C was more toxic than free $\mathrm{D}-\mathrm{C}$ on both cell lines. Due to the conjugation of the targeting ligand to PEGNIO/D-C, PEGNIO/D-C/t-Lyp-1 was more toxic to U87 cells than to hMSC cells after $24 \mathrm{~h}(p<$ 0.05). PEGNIO/D-C/t-Lyp-1 significantly increased the cytotoxicity for U87 cells in comparison to free $\mathrm{D}-\mathrm{C}(p<0.05)$. Moreover, U87 cells were treated with samples (D-C, PEGNIO/D-C and PEGNIO/ D-C/t-Lyp-1) for $48 \mathrm{~h}$ at concentration range of doxorubicin (0-50 $\left.\mu \mathrm{g} \mathrm{mL}{ }^{-1}\right)$. $\mathrm{IC}_{50}$ values were calculated as to be $0.96 \mu \mathrm{g} \mathrm{mL} \mathrm{m}^{-1}, 0.9$ $\mu \mathrm{g} \mathrm{mL} \mathrm{mL}^{-1}$ and $0.76 \mu \mathrm{g} \mathrm{mL} \mathrm{m}^{-1}$ for D-C, PEGNIO/D-C and PEGNIO/ D-C/t-Lyp-1 respectively.

\section{Effect on tumor-like multicellular spheroids}

According to results obtained in $2 \mathrm{D}$ cell cultures, it is clear that the tLyp- 1 conjugated niosomal formulation acted as a targeted multi-drug delivery platform for NRP-1 expressing tumor cells. Further, we have used 3D spheroid model which is found to be relevant for therapeutic evaluation, as it reflects better the in vivo conditions both in structural and molecular aspects. ${ }^{34}$ The effect on tumor-like spheroids was evaluated following the treatment with cell culture media, free D-C, PEGNIO/D-C and PEGNIO/D-C/tLyp-1 for 6 days. As shown in Fig. 4a, the tumor spheroids treated with cell culture media retained their morphology (compact spheroids with smooth surface) over entire period of cultivation. The spheroids treated with D-C became smaller in diameter (Fig. 4b). The tumor spheroids exposed to niosomal formulations exhibited no more tightly organised structure (Fig. 4c and d). Especially, PEGNIO/D-C/ tLyp-1 treated spheroids became distorted, with many
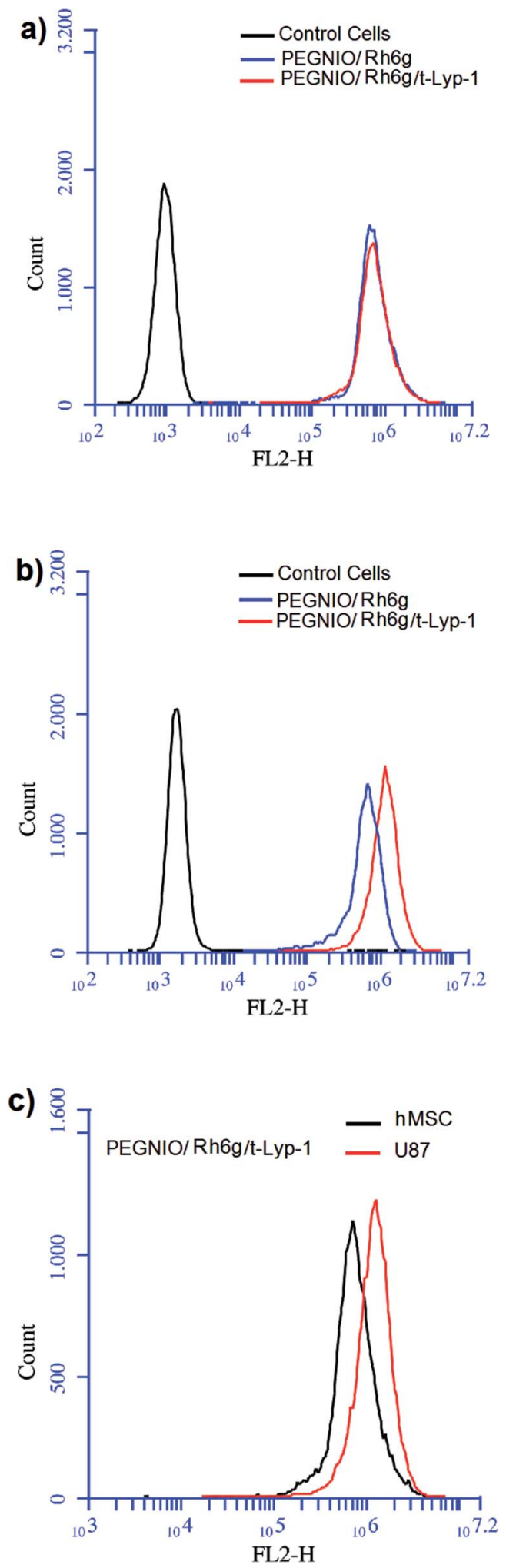

Fig. 2 Flow cytometry measurements of Rh6g uptake by hMSC (a) and U87 cells (b) after incubating with PEGNIO/Rh6g and PEGNIO/Rh6g/ t-Lyp-1. Histogram of binding of PEGNIO/Rh6g/t-Lyp-1 to hMSC and U87 cells (c)

disassociated cells (Fig. 4d). These results demonstrated enhanced effects of PEGNIO/D-C/tLyp-1 on 3D tumor-like tissues in compare to other formulations. 


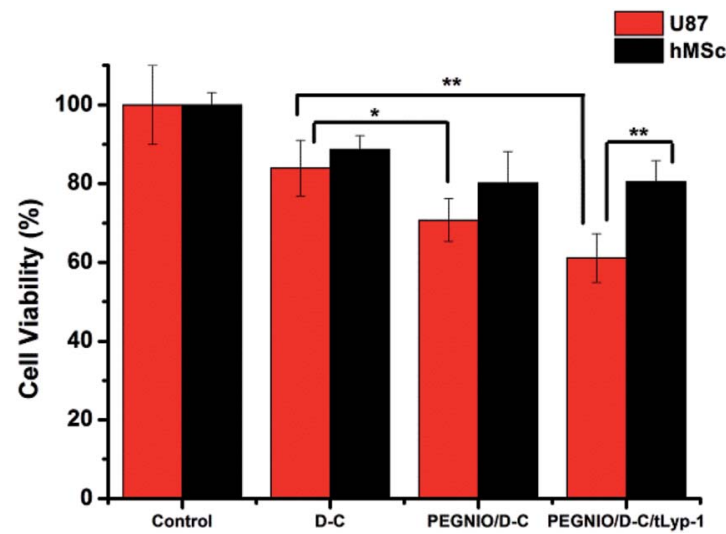

Fig. 3 Cytotoxicity of the free drug and niosomal formulations on hMSC and U87 cells. Cells were incubated with, PEGNIO/D-C, PEGNIO/D-C/t-Lyp-1 and free D-C (equivalent concentration of loaded D-C) for $24 \mathrm{~h}$. MTT assay was applied. Error bars represent the standard deviation from the mean $(N=3)$. Data were analyzed using $t$ test, and $* p<0.05, * * p<0.01$ was considered significant and very significant respectively.

a)

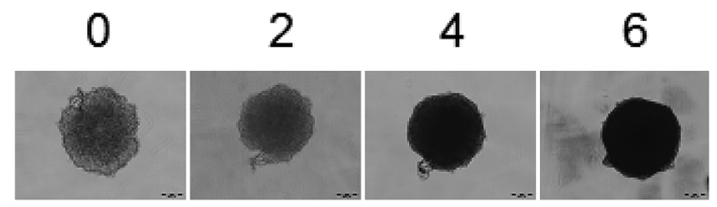

b)

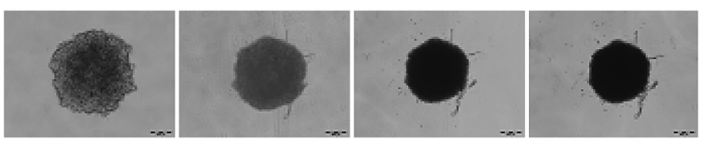

c)

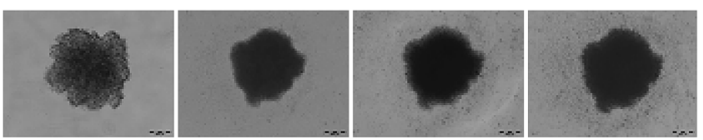

d)

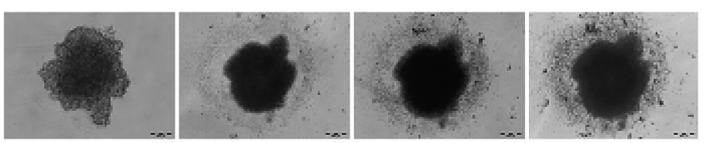

Fig. 4 Morphology of U87 tumor spheroids treated with cell culture media (a), D-C (b), PEGNIO/D-C (c) and PEGNIO/D-C/t-Lyp-1 (d) on days $0,2,4$ and 6 , respectively.

\section{Conclusion}

In this work, a targeted niosomal co-drug delivery system was developed to improve the therapeutic efficacy of anti-glioma drug delivery. Curcumin and doxorubicin were encapsulated into PEGNIO by the thin film hydration method. The surface of PEGNIO was decorated with tLyp-1, which is tumor homing and penetrating peptide. Tumor-like structure dissociation in 3D tumor spheroids, enhanced cellular interaction, and increased cytotoxicity of the drugs in U87 cells were achieved by PEGNIO/ D-C/tLyp-1. These data indicated that the formulation PEGNIO/ D-C/tLyp-1 might be a promising and efficient strategy for drug delivery in anti-glioma therapy.

\section{Acknowledgements}

The Deutsche Forschungsgemeinschaft (DFG) within the REBIRTH cluster of excellence funded part of this work. Konrad Adenauer Foundation is acknowledged for the financial support to Didem Ag Seleci. We wish to thank André Jochums for his help with flow cytometry analysis. Special thank to Rebecca Jonczyk and Johanna-Gabriela Walter for technical checking of the manuscript. The publication of this paper was funded by the Open Access Fund of the Leibniz Universitat Hannover.

\section{Notes and references}

1 X. Wei, X. Chen, M. Ying and W. Lu, Acta Pharm. Sin. B, 2014, 4, 193-201.

2 B. Mujokoro, M. Adabi, E. Sadroddiny, M. Adabi and M. Khosravani, Mater. Sci. Eng., C, 2016, 69, 1092-1102.

3 B. Al-Lazikani, U. Banerji and P. Workman, Nat. Biotechnol, 2012, 30, 679-692.

4 Q. Hu, G. Gu, Z. Liu, M. Jiang, T. Kang, D. Miao, Y. Tu, Z. Pang, Q. Song and L. Yao, Biomaterials, 2013, 34, 11351145.

5 D. Miao, M. Jiang, Z. Liu, G. Gu, Q. Hu, T. Kang, Q. Song, L. Yao, W. Li and X. Gao, Mol. Pharmaceutics, 2013, 11, 90101.

6 F. Dilnawaz and S. K. Sahoo, Eur. J. Pharm. Biopharm., 2013, 85, 452-462.

7 Y. Cui, Q. Xu, P. K.-H. Chow, D. Wang and C.-H. Wang, Biomaterials, 2013, 34, 8511-8520.

$8 \mathrm{Y}$. Xu, M. Shen, Y. Sun, P. Gao and Y. Duan, J. Nanosci. Nanotechnol., 2015, 15, 9777-9787.

9 A. Sankhyan and P. Pawar, J. Appl. Pharm. Sci., 2012, 02, 2032.

10 D. Pando, M. Matos, G. Gutiérrez and C. Pazos, Colloids Surf., $B, 2015,128,398-404$.

11 L. Tavano, R. Muzzalupo, N. Picci and B. de Cindio, Colloids Surf., B, 2014, 114, 144-149.

12 L. Tavano, L. Gentile, C. O. Rossi and R. Muzzalupo, Colloids Surf., B, 2013, 110, 281-288.

13 D. Ag Seleci, M. Seleci, J.-G. Walter, F. Stahl and T. Scheper, J. Nanomater., 2016, 2016, 7372306.

14 L. Tavano, L. Mauro, G. D. Naimo, L. Bruno, N. Picci, S. Ando and R. Muzzalupo, Langmuir, 2016, 32, 8926-8933.

15 P. Li and T. G. Rossman, Toxicol. Sci., 2001, 64, 90-99.

16 C. Nasarre, M. Roth, L. Jacob, L. Roth, E. Koncina, A. Thien, G. Labourdette, P. Poulet, P. Hubert and G. Cremel, Oncogene, 2010, 29, 2381-2392.

17 Q. Hu, X. Gao, G. Gu, T. Kang, Y. Tu, Z. Liu, Q. Song, L. Yao, Z. Pang and X. Jiang, Biomaterials, 2013, 34, 5640-5650.

18 E. Ruoslahti, Adv. Mater., 2012, 24, 3747-3756.

19 L. Roth, L. Agemy, V. R. Kotamraju, G. Braun, T. Teesalu, K. N. Sugahara, J. Hamzah and E. Ruoslahti, Oncogene, 2012, 31, 3754-3763.

20 B. Xu, Y. Ju, G. Song and Y. Cui, J. Nanopart. Res., 2013, 15, 2105. 
21 M. N. Azmin, A. T. Florence, R. M. Handjani Vila, J. F. B. Stuart, G. Vanlerberghe and J. S. Whittaker, J. Pharm. Pharmacol., 1985, 37, 237-242.

22 D. A. Seleci, M. Seleci, A. Jochums, J.-G. Walter, F. Stahl and T. Scheper, $R S C$ Adv., 2016, 6, 87910-87918.

23 L. Tavano, R. Muzzalupo, L. Mauro, M. Pellegrino, S. Ando and N. Picci, Langmuir, 2013, 29, 12638-12646.

24 R. Nallamothu, G. C. Wood, C. B. Pattillo, R. C. Scott, M. F. Kiani, B. M. Moore and L. A. Thoma, AAPS PharmSciTech, 2006, 7, E7-E16.

25 Q. Hu, X. Gao, T. Kang, X. Feng, D. Jiang, Y. Tu, Q. Song, L. Yao, X. Jiang and H. Chen, Biomaterials, 2013, 34, 94969508.

26 M. Tan, J. Luo and Y. Tian, RSC Adv., 2014, 4, 61948-61959.

27 L. Tavano, R. Aiello, G. Ioele, N. Picci and R. Muzzalupo, Colloids Surf., B, 2014, 118, 7-13.
28 B. Xu, Y. Ju, Y. Cui, G. Song, Y. Iwase, A. Hosoi and Y. Morita, Langmuir, 2014, 30, 7789-7797.

29 L. Sun, X. Deng, X. Yang, Z. Li, Z. Wang, L. Li, Q. Wu, F. Peng, L. Liu and C. Gong, RSC Adv., 2014, 4, 46737-46750.

30 X. Zhao, Q. Chen, Y. Li, H. Tang, W. Liu and X. Yang, Eur. J. Pharm. Biopharm., 2015, 93, 27-36.

31 M. Singh and N. Singh, Mol. Cell. Biochem., 2011, 347, 1-11. 32 N. Dhillon, B. B. Aggarwal, R. A. Newman, R. A. Wolff, A. B. Kunnumakkara, J. L. Abbruzzese, C. S. Ng, V. Badmaev and R. Kurzrock, Clin. Cancer Res., 2008, 14, 4491-4499.

33 Y. G. Lin, A. B. Kunnumakkara, A. Nair, W. M. Merritt, L. Y. Han, G. N. Armaiz-Pena, A. A. Kamat, W. A. Spannuth, D. M. Gershenson and S. K. Lutgendorf, Clin. Cancer Res., 2007, 13, 3423-3430.

34 J. Friedrich, C. Seidel, R. Ebner and L. A. Kunz-Schughart, Nat. Protoc., 2009, 4, 309-324. 